\title{
Philosophiques
}

\section{Le problème mathématique de l'espace : à propos d'un ouvrage récent de Luciano Boi - présentation}

\section{Robert Nadeau}

Volume 24, numéro 2, automne 1997

URI : https://id.erudit.org/iderudit/027457ar

DOI : https://doi.org/10.7202/027457ar

Aller au sommaire du numéro

Éditeur(s)

Société de philosophie du Québec

ISSN

0316-2923 (imprimé)

1492-1391 (numérique)

Découvrir la revue

Citer ce document

Nadeau, R. (1997). Le problème mathématique de l'espace : à propos d'un ouvrage récent de Luciano Boi - présentation. Philosophiques, 24(2), 345-347. https://doi.org/10.7202/027457ar d'utilisation que vous pouvez consulter en ligne. 


\title{
TABLE RONDE
}

\section{IE PROBLÈME MATHÉMATIQUE DE L'ESPACE À PROPOS D'UN OUVRAGE RÉCENI DE LUCIANO BOI}

\author{
PRÉSENTATION \\ PAR \\ Robert Nadeau
}

Le mercredi 15 mai 1996, dans le cadre du congrès annuel de la Société de Philosophie du Québec (ACFAS) qui se tenait à l'Université McGill, eut lieu une table ronde consacrée à l'examen d'un ouvrage récent de Luciano Boi. Les tenants et les aboutissants de ce brillant ouvrage, intitulé Le problème mathématique de l'espace. Une quête de l'intelligible (préfacé par René Thom, Heidelberg/Berlin, Springer, 1995, 115 illustrations, 526 p.) furent successivement analysés par Liliane Beaulieu (Centre de Recherches Mathématiques, Univ. de Montréal, et Département de philosophie, Collège de Rosemont), François Lalonde (Département de mathématique, UQAM, et directeur de l'Institut de Sciences Mathématiques) Alain Michel (Département de philosophie, Université de Provence, Aix-en-Provence), et enfin Jean Petitot (Centre d'Analyse et de Mathématique Sociales, École des Hautes Études en Sciences Sociales, Paris). On trouvera ici le texte de ces deux dernières études critiques, suivi de la réplique de Luciano Boi.

Je rappelle, pour mémoire, que cette table ronde fut suivie, trois jours plus tard à l'UQAM, d'un colloque d'une journée consacré aux a Nouvelles perspectives sur les mathématiques et la physique modernes n, qui permit de réunir comme conférenciers Jean Petitot, Alain Michel, Luciano Boi et Pierre Kerszberg (Département de philosophie, Pennsylvania State University).

La jeune tradition des tables rondes de la SPQ à l'ACFAS permet de saluer quelques-unes des publications philosophiques remarquables de l'année qui précède. En 1995, le congrès de Chicoutimi avait donné lieu à l'organisation de trois telles tables rondes (la première autour de William Shea et portant sur la carrière scientifique de Descartes ; la seconde autour de François Duchesneau et concernant l'invention de la dynamique par 
Leibniz ; la troisième, enfin, autour de Storrs McCall et portant sur le modèle de l'univers qui se dégage de la physique contemporaine). Tous les textes qui furent présentés à l'époque ont depuis lors fait l'objet d'une publication dans la revue Philosophiques (voir « La philosophie des sciences au Québec : trois contributions majeures ", Philosophiques, vol. XXII, N², automne 1995, sous la rubrique Tables rondes, p. 337-509).

Je dirai maintenant quelques mots au sujet des participants à la table ronde du 15 mai 1996. Concernant Luciano Boi d'abord. Au cours des années récentes et en particulier au cours des années pendant lesquelles il préparait l'ouvrage dont il sera question ici, Luciano Boi a séjourné au Centre d'Analyse et de Mathématiques Sociales ainsi qu'au Centre Alexandre Koyré de l'École des Hautes en Sciences Sociales de Paris. Il a en outre séjourné durant trois ans à l'Institut für Philosophie, Wissenschaftstheorie, Wissenschaftsund Technikgeschichte, de la Technische Universität de Berlin, soit de mai 1991 à mai 1994, une information qui n'est pas sans intérêt compte tenu que l'ouvrage sous examen dans les pages qui suivent a été rédigé à compter de septembre 1991.

Je dirai peu de chose pour présenter l'ouvrage de Luciano Boi, deux spécialistes le feront beaucoup mieux que moi plus bas. Mais, globalement, quel est l'objectif de L. Boi dans cet ouvrage? Comme le précise René Thom dans sa préface, dans laquelle Thom se livre à ce qu'il appelle une archéologie de la géométrie euclidienne ", l'auteur de l'ouvrage à l'étude ici se préoccupe “ du long procès conceptuel qui a permis de s'évader du " carcan " de la géométrie euclidienne classique imposée au curriculum des études scientifiques jusqu'à l'époque moderne "(ibid., p. vii). Ce livre récapitule en fait l'histoire des concepts mathématiques essentiels apparus au cours du XIX ${ }^{e}$ siècle.

Comme le souligne lui-même Luciano Boi dans son Avant-propos, " [L]'invention des géométries non euclidiennes a représenté une rupture conceptuelle décisive dans la pensée mathématique, comparable en importance à la découverte du calcul infinitésimal et intégral aux XVII et $\mathrm{XVIII}^{\mathrm{e}}$ siècles. Elle a ainsi considérablement transformé le paysage des mathématiques et la conception que l'on pouvait en avoir n(ibid., p. xiv).

L'ouvrage de L. Boi est divisé en trois parties. La première est consacrée à la découverte de la géométrie non euclidienne et à l'impact de cette découverte sur l'édifice global des mathématiques, qui en entraîne la "métamorphose", selon l'expression de L. Boi. La deuxième partie est consacrée à l'examen de la notion de variété, qui apparaît avec les travaux de Bernhard Riemann. Enfin, la troisième partie examine la contribution de Beltrami (géométrie pseudosphérique), de Helhmholtz et de Clifford (géométrie elliptique) à la mise au point d'une géométrie que L. Boi qualifie d' đ infinitésimale intrinsèque, projective et non euclidienne ». 
Le premier discutant de l'ouvrage de L. Boi est Alain Michel, directeur du département de philosophie de l'Université de Provence (Aix-enProvence). A. Michel est spécialiste de la philosophie des mathématiques.

Le second discutant est Jean Petitot, chercheur rattaché au Centre d'Analyse et de Mathématique Sociales de l'École des Hautes Études en Sciences Sociales de Paris. J. Petitot est également chercheur associé au Centre de Recherche en Épistémologie Appliquée (CRÉA, École Polytechnique, Paris). Ses travaux sont aussi bien ceux d'un mathématicien que ceux d'un philosophe, et ses centres d'intérêt sont multiples, ce qui peut avoir quelque chose de tout à fait déroutant pour un observateur extérieur, puisqu'ils concernent aussi bien la physique et les mathématiques modernes que la philosophie transcendantale de Kant, la sémiologie littéraire, les sciences cognitives.

Il a été convenu que, sans nécessairement viser à faire le tour complet de toutes les questions abordées dans cet ouvrage complexe, chacun des discutants en ferait un examen dans sa perspective propre, et que l'auteur de l'ouvrage aurait après coup, comme il se doit, un droit de réplique. Ce sont précisément ces trois textes qu'on pourra maintenant lire dans les pages qui suivent.

Département de philosophie

Université du Québec à Montréal 


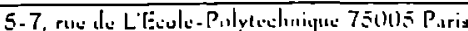

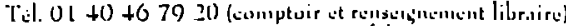

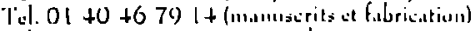

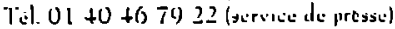

Tí. $01+0$ t6 79 ? I (dirction sumbercisle)

Fox OL $+329 \leqslant 020$ (in.tunserits, fulbrication)

Fux $01+3258203$ (eummercinl)
Josiane BOULAD-AYOUB

m.s.r.c.

Michel GRENON

Édition nouvelle

présentée. mise à jour et augmentée

des

\title{
PROCÈS-VERBAUX DU COMITÉ D'INSTRUCTION PUBLIQUE DE L'ASSEMBLÉE LÉGISLATIVE ET DE LA CONVENTION NATIONALE
}

\author{
Préface de Michel VOVELLE
}

En 1889, le centenaire de la Révolution donna lieu à la publication des Procès-verbaıtr du Comité d'Instruction publique de l'Assemblée législative, édité par les soins de I'historien James Guillaume. Huit lourds volumes suivirent jusqu'en 1907 pour former les Procès-verbaux du Comité d'Instruction publique de la Convention nationale. Ils sont devenus pratiquement introuvables.

Composés de procès-verbaux des séances, chacune accompagnée en annexe des rapports du Comité, des projets de décrets, de pétitions rédigées par divers corps de citoyens et d'extraits de journaux du temps, ils constituent un instrument de travail indispensable dans le domaine de l'histoire intellectuelle, phılosophique, scientilique et culturelle des Lumières et de la Révolution.

Cente nouvelle édition est enrichie dans son premier volume d'une préface de Michel Vovelle, d'une introduction générale au travail de Guillaume et à ses enjeux ainsi que d'une bio-bibliographe. Enfin, s'y ajoutent des documents des pénodes de la Constituante et du Directoire, non couventes par Guillaume. Pour plus de maniabilité chacun des volumes comprend deux fascicules, le deuxième comportant les nores, commentaires et tables analytiques de matière. Le tout est vendu sous la torme d'un coltiret.

J. Boulad-A youb, professeur au département de philosophie et $M$. Grenon, professeur au département d'hıstoire, tous deux de l'Université de Québec à Montréal, dirigent une équipe interdisciplinaire s'intéressant à la philosophie, à l'histoire et à la culture de l'Europe des XVIII" et XIX' ${ }^{\mathrm{i}}$ siècles.

ISBN, vol. Ià IX: 2-738+5790-8; 2.738- 579l-6; 2-738+-5792-4: 2-738--579-1-0; 2-738+-5795-9; 2-738+-580 I-7: 2.738+5802-5, 2-738- $5803-3 ;$ 2-738- -5804-

\section{BO.N DE CONI.LA.VDE}

Á recoumer à L'Harmallan, 7, rue de l'École-Polylechnique, 75005 Pars

Veuillez me faire parveair exemplaire (s) de

Procès-verbaict du Contlé d'insiruction publique de l'Assemblée législative el de la Convention narionale édicion revisée ec augmentée de J. Boulad-Ayoub et $\$$. Grenon, le coltret au prix unutaire

de : $3000 \mathrm{FF}$ plus $120 \mathrm{FF}$ de port, soil un total de $3120 \mathrm{FF}$

NOMI

\section{ADRESSE}

Ci-joint un chèque de

$\mathrm{FF}$

- Pour l'éranger vos règlements sont à elfectuer

- En trancs trançaus sur chíque domicilić sur banque françusc

- Par virement en irancs liranças sur noure CCP 2362 jH XP Pans 